\title{
Treatment-emergent neuroendocrine prostate cancer with a germline BRCA2 mutation: identification of a candidate reversion mutation associated with platinum/PARP-inhibitor resistance
}

\author{
Deep Pandya, ${ }^{1,6}$ Myra Shah, ${ }^{2,6}$ Fuat Kaplan, ${ }^{1}$ Candice Martino, ${ }^{1}$ Gillian Levy, ${ }^{3}$ \\ Mia Kazanjian, ${ }^{4,5}$ Stephen Batter, ${ }^{5}$ John Martignetti, $^{1}$ and Richard C. Frank ${ }^{1,2}$ \\ ${ }^{1}$ Rudy L. Ruggles Biomedical Research Institute, Nuvance Health, Danbury, Connecticut 06810, USA; \\ ${ }^{2}$ Department of Medicine, ${ }^{3}$ Department of Pathology, ${ }^{4}$ Department of Radiology, ${ }^{5}$ Department of Urology, \\ Norwalk Hospital, Nuvance Health, Norwalk, Connecticut 06856, USA
}

\begin{abstract}
Neuroendocrine prostate cancer (NEPC) is a highly aggressive histologic subtype of prostate cancer associated with a poor prognosis. Its incidence is expected to increase as castration-resistant disease emerges from the widespread use of potent androgen receptortargeting therapies, such as abiraterone and enzalutamide. Defects in homologous recombination repair genes, such as BRCA $1 / 2$, are also being increasingly detected in individuals with advanced prostate cancer. We present the case of a 65 -yr-old man with a germline BRCA2 mutation who developed explosive treatment-emergent, small-cell neuroendocrine prostate cancer. He achieved a complete response to platinum-containing chemotherapy, but a limited remission duration with the use of olaparib, a poly(ADP-ribose) polymerase (PARP) inhibitor, as maintenance therapy. Upon relapse, tumor genomic profiling revealed a novel 228-bp deletion in exon 11 of the BRCA2 gene. The addition of the anti-PD1 drug pembrolizumab to olaparib was ineffective. This case highlights the ongoing challenges in treating neuroendocrine prostate cancer, even in the setting of homologous recombination repair deficiency.
\end{abstract}

Corresponding author: richard.frank@nuvancehealth.org

(C) 2021 Pandya et al. This article is distributed under the terms of the Creative Commons Attribution-NonCommercial License, which permits reuse and redistribution, except for commercial purposes, provided that the original author and source are credited.

Ontology term: prostate cancer

Published by Cold Spring Harbor Laboratory Press

doi:10.1101/mcs.a005801
[Supplemental material is available for this article.]

\section{INTRODUCTION}

Metastatic castration-sensitive prostate cancer is typically treated in the first-line setting with androgen deprivation therapy in combination with either the chemotherapeutic drug docetaxel or a newer, orally active hormonal agent, such as abiraterone or enzalutamide (Swami et al. 2020). Treatment resistance, however, is universal and associated with transdifferentiation to a small-cell neuroendocrine histology in 17\% of cases (Aggarwal et al. 2018). Treatment-emergent small-cell neuroendocrine prostate cancer (t-SCNC) has few effective therapeutic options. Platinum-based chemotherapy is typically used as first-line therapy, although median survivals are only $7 \mathrm{mo}$ (Wang et al. 2014). Improvements in the treatment of t-SCNC are, therefore, urgently needed.

\footnotetext{
${ }^{6}$ These authors contributed equally to this work.
} 
COLD SPRING HARBOR Molecular Case Studies
Neuroendocrine prostate cancer with BRCA mutation
Germline ( $g$ ) and somatic mutations in homologous recombination repair (HRR) genessuch as BRCA1/2, ATM, PALB2, RAD51D, and CHEK2-are estimated to occur in $12 \%$ and $25 \%$ of individuals with castrate-resistant prostate cancer (CRPC), respectively (Sartor and de Bono 2018). Although the superiority of platinum-based chemotherapy has not been established in this setting (Mateo et al. 2018), as it has for other types of HRR-deficient adenocarcinomas (Robson et al. 2017; Moore et al. 2018; Golan et al. 2019), the effectiveness of poly (ADP-ribose) polymerase (PARP) inhibitors has been proven (de Bono et al. 2020). In contrast, neuroendocrine prostate cancer has demonstrated sensitivity to platinum agents (Apostolidis et al. 2019; Corn et al. 2019), but there are no clinical trial reports on the use of PARP inhibitors in this setting. Given the expanding linkage between platinum sensitivity and responsiveness to PARP inhibiton in malignancies characterized by HRR deficiency, it is reasonable to study this treatment paradigm in patients with HRR-deficient t-SCNC.

Here, we report the case of a man with an aggressive t-SCNC carrying a gBRCA2 mutation who was treated with the PARP inhibitor (PARPi) olaparib following a major response to first-line platinum-based chemotherapy.

\section{RESULTS}

\section{Clinical Presentation and Family History}

Informed written consent was obtained from the patient for publication of this case report. A 65-yr-old Indian male, with a history of type 1 diabetes, presented to the Norwalk Hospital emergency department after 1 mo of progressive bilateral leg and scrotal edema as well as low back pain. Ultrasound showed an occlusive deep vein thrombosis (DVT) in the left common femoral vein with possible extension into the left external iliac vein. He underwent thrombectomy followed by treatment with enoxaparin. Computed tomography (CT) of the chest, abdomen, and pelvis showed thrombus in the left iliac veins, multiple enlarged retroperitoneal and left pelvic lymph nodes, and prostatic enlargement, measuring $5.1 \times 5.3 \times 5.3$ $\mathrm{cm}$. A bone scan showed several widely distributed osseous metastases involving the hips, spine, ribs, and sternum. The prostate-specific antigen (PSA) was $95 \mathrm{ng} / \mathrm{mL}$. He underwent 12-quadrant prostate biopsies, which showed $50 \%-100 \%$ involvement in all cores with Gleason $8(4+4)$ adenocarcinoma. The tumor was strongly positive for prostate-specific acid phosphatase (Fig. 1).

The patient began leuprolide androgen deprivation therapy (ADT) and abiraterone plus prednisone, choosing to avoid chemotherapy because of the potential impact of side effects on his employment. His initial symptoms gradually improved and he was soon able to return to work and carry out all activities of daily living (ADLs). The PSA declined to $0.2 \mathrm{ng} / \mathrm{mL}$ during the first $3 \mathrm{mo}$ and remained low, as shown in Figure 2. After $12 \mathrm{mo}$, a bone scan showed decreased intensity and extent of all lesions, and CT showed no visceral disease (Fig. 2). Germline genetic testing revealed a pathogenic nonsense mutation in the BRCA2 gene, predicting a truncated protein (Table 1 and next section). Family history was notable for a paternal uncle with prostate cancer and a maternal uncle with colon cancer, both in their 60s.

After $16 \mathrm{mo}$, the patient became acutely ill requiring hospitalization. Physical examination revealed scleral icterus, painful hepatomegaly, and profound global weakness. The aspartate transaminase was $424 \mathrm{U} / \mathrm{L}$, alanine transaminase $241 \mathrm{U} / \mathrm{L}$, total bilirubin $5.4 \mathrm{mg} / \mathrm{dL}$, and lactate $5.0 \mathrm{mmol} / \mathrm{L}$. The PSA was $0.5 \mathrm{ng} / \mathrm{mL}$, serum chromogranin-A $7160 \mathrm{ng} / \mathrm{mL}$ (nor$\mathrm{mal}<95$ ), and neuron-specific enolase (NSE) $824 \mathrm{ng} / \mathrm{mL}$ (normal < 15). Abdominopelvic CT showed the liver was $27 \mathrm{~cm}$ in transverse dimension with innumerable new 2-3 cm hypodense lesions (Fig. 2). CT-guided liver biopsy showed poorly differentiated neuroendocrine carcinoma most consistent with small-cell carcinoma (Fig. 1). Immunohistochemical stains showed the tumor positive for CK20 (dot-like accentuation), synaptophysin, and 

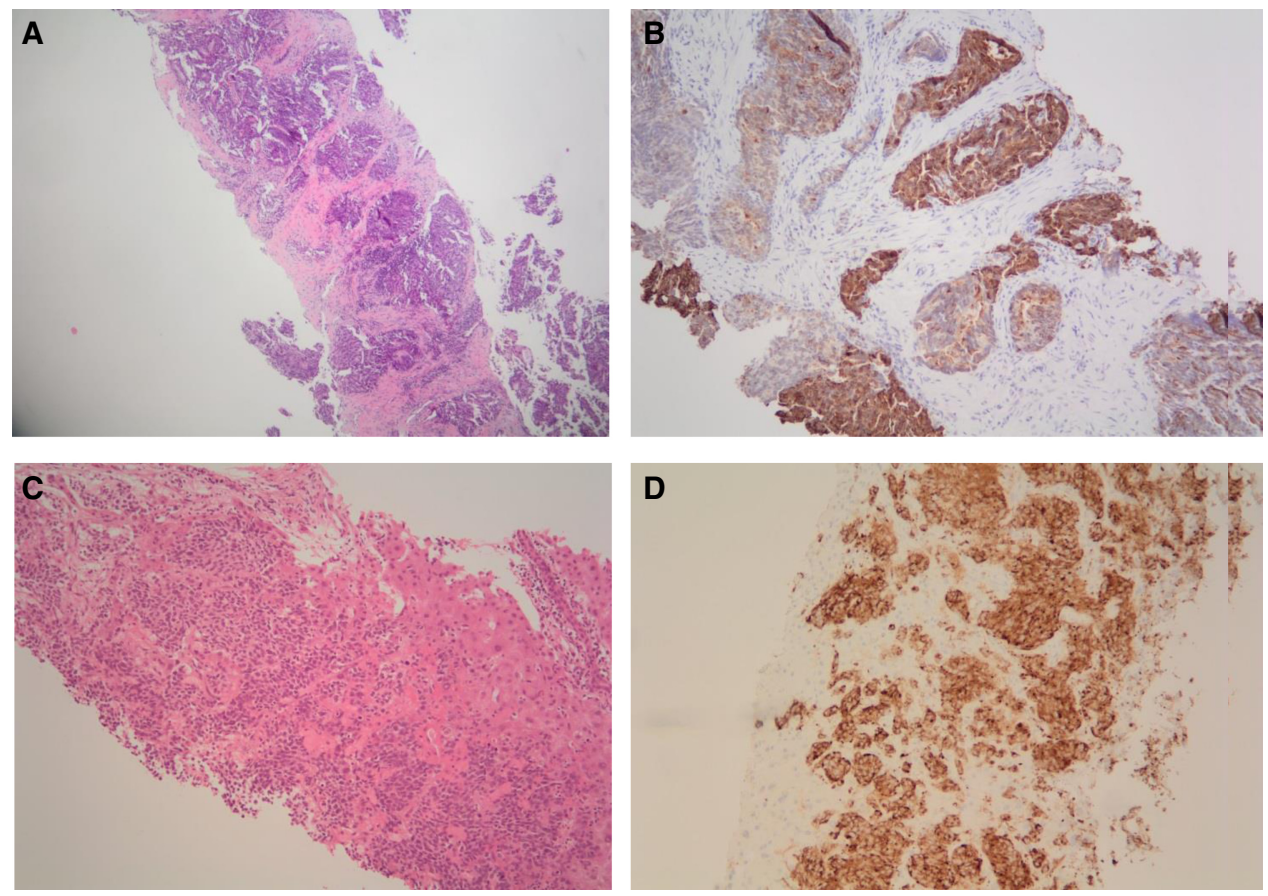

Figure 1. (A) Prostate adenocarcinoma: low-power view from left lateral mid prostate biopsy showing Gleason $4+4=8$ pattern $(4 \times)$. (B) Prostatic specific acid phosphatase (PSAP): low-power view from left lateral mid prostate biopsy showing positive cytoplasmic staining for PSAP by immunohistochemistry (IHC) (10x). (C) Liver small-cell carcinoma: low-power view of tumor in the liver with nests and sheets of uniform tumor cells with high nuclear to cytoplasmic ratio $(10 \times)$. (D) Liver synaptophysin: IHC for synaptophysin showing membranous and cytoplasmic positive staining (20x).

chromogranin and negative for CK7, PSA, TTF-1, GATA-3, CDX-2, NKX3.1, and Merkel cell polyoma virus. There was loss of RB and the Ki-67 labeling index was $>50 \%$. There was insufficient tumor sample for genomic profiling prechemotherapy but this was performed subsequently (see below).

The patient began chemotherapy with etoposide and carboplatin every $3 \mathrm{wk}$ and gradually improved with normalization of liver enzymes. After two cycles of treatment, the NSE declined to $18 \mathrm{ng} / \mathrm{mL}$, chromogranin-A declined to $408 \mathrm{ng} / \mathrm{mL}$, and CT scan showed markedly diminished hepatic metastases. After six cycles of chemotherapy, the chromogranin-A was $115 \mathrm{ng} / \mathrm{mL}$, and CT showed near-complete resolution of hepatic metastases (Fig. 2). The patient was then placed on maintenance therapy with the PARPi olaparib (300 mg po bid). Olaparib was well-tolerated without significant side effects, but within 6 mo of treatment, CT scan showed recurrent hepatic metastases (Fig. 2). Upon the development of PARP inhibitor resistance, cell-free (cf) DNA analysis revealed only the pathogenic germline BRCA2 mutant. Genomic profiling from a growing hepatic metastasis, however, revealed a 228-bp deletion in BRCA2 that restored the open reading frame. This mutation results in near full-length BRCA2 protein and would be considered a reversion mutation (see the next section).

Because of the lack of known effective therapies for refractory t-SCNC, the patient was next treated with pembolizumab (200 mg every $3 \mathrm{wk}$ ) while olaparib was continued, given the preliminary efficacy of anti-PD1 immunotherapy drugs in patients with CRPC and an HRR gene mutation (Antonarakis et al. 2020). After three cycles of therapy, continued progression of disease was found by imaging and tumor markers (Fig. 2), and the patient began to lose weight and experience hepatic pain. He was again treated with platinum-based 

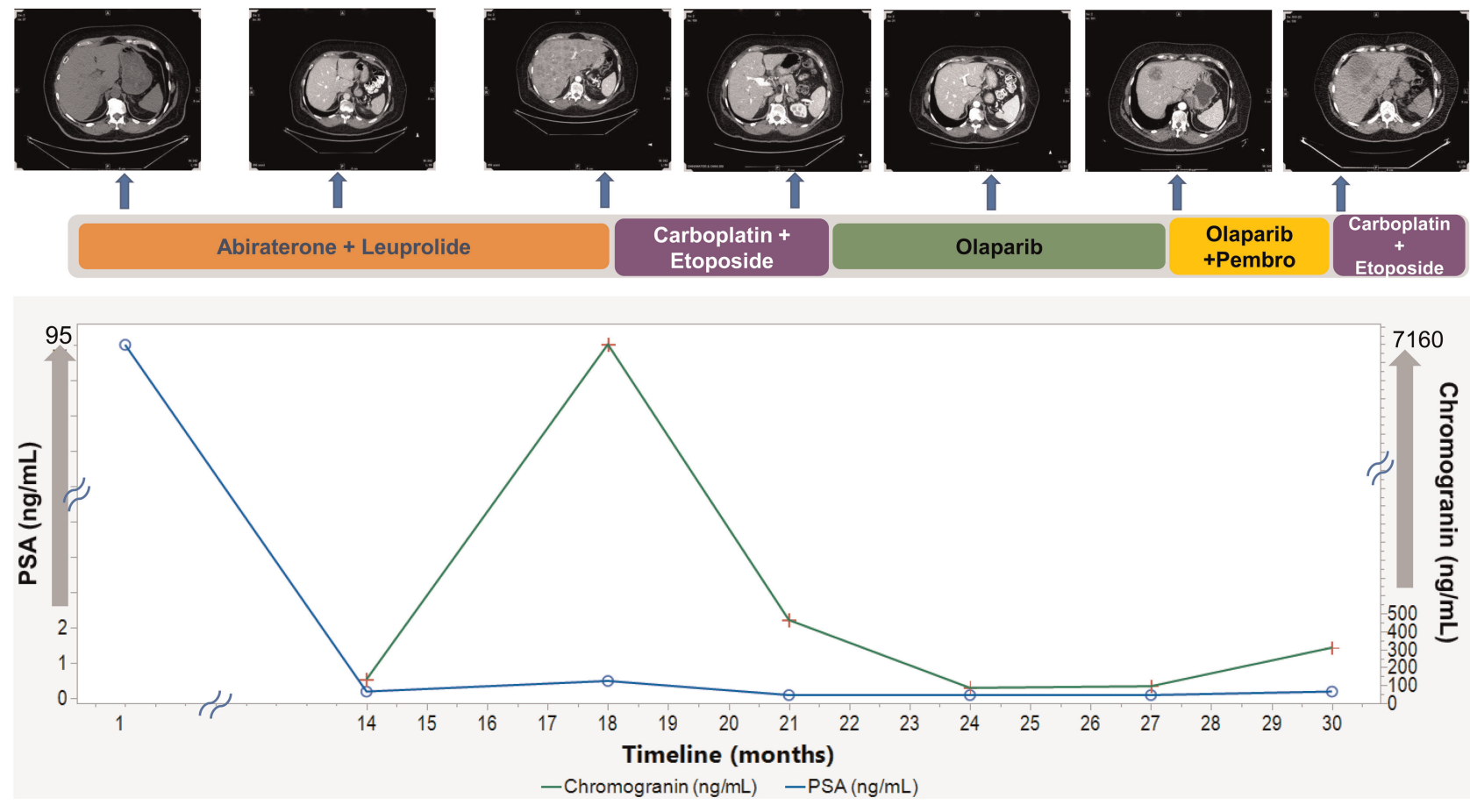

Figure 2. Clinical pattern of response to treatment. PSA and Chromogranin A measurement levels corresponding to the timeline showing therapy (middle panel). Top panels show corresponding axial CT images. N.B. Leuprolide continued but abiraterone was discontinued upon diagnosis of neuroendocrine carcinoma. (Pembro) Pembrolizumab.

chemotherapy, but after three cycles developed global progression of disease including innumerable brain metastases. Hospice care was chosen and he died 18 mo after the diagnosis of t-SCNC and 34 mo from diagnosis of metastatic prostate cancer.

\section{Germline and Somatic Genomic Alterations}

Germline genetic testing was performed prior to initiation of chemotherapy by the 30-gene Color Hereditary Cancer Risk Test (Color Genomics). This revealed the presence of a pathogenic BRCA2 nonsense mutation (p.Ser1882*) at a mutation allele frequency (MAF) of $49.3 \%$, which predicts truncation and loss of full-length functional protein. A variant of uncertain significance (VUS) in the ATM gene (p.12185T) was also found (Table 1).

Later during the course of treatment and in the setting of resistance to PARP inhibitor therapy, blood for cfDNA was analyzed on the Guardant360 platform consisting of 74 cancer-associated genes (Guardant Health), revealing the same germline BRCA2 mutation with a MAF of $46.9 \%$ and a somatic BRCA1 (p.E255D) VUS with a MAF of $6.9 \%$ (Table 1). Liver biopsy confirmed SCNC and genomic profiling was performed on the MSK-IMPACT platform consisting of 468 genes (Memorial Hospital For Cancer \& Allied Diseases) (Zehir et al. 2017). This revealed the following alterations; a somatic BRCA2 mutation ( $p$. V1810_C1885del) with a MAF of 56\%, a VUS in BRCA1, benign ALK, HGF, and likely pathogenic SETD2 mutations (Table 1). Copy-number alterations (CNAa): PTEN, TNRFSF14, HNF1A, PIK3CD, PDCD1, and SETD8. CNA from these genes are suggestive of multiple chromosomal level losses and deletion of the entire gene PTEN (Table 2). Structural variants: MAP2K1, KMT2C2, and TMPRSS2-ERG fusion (Table 2), supporting the transdifferentiation of the small-cell neuroendocrine cancer from the original prostate adenocarcinoma, as this 


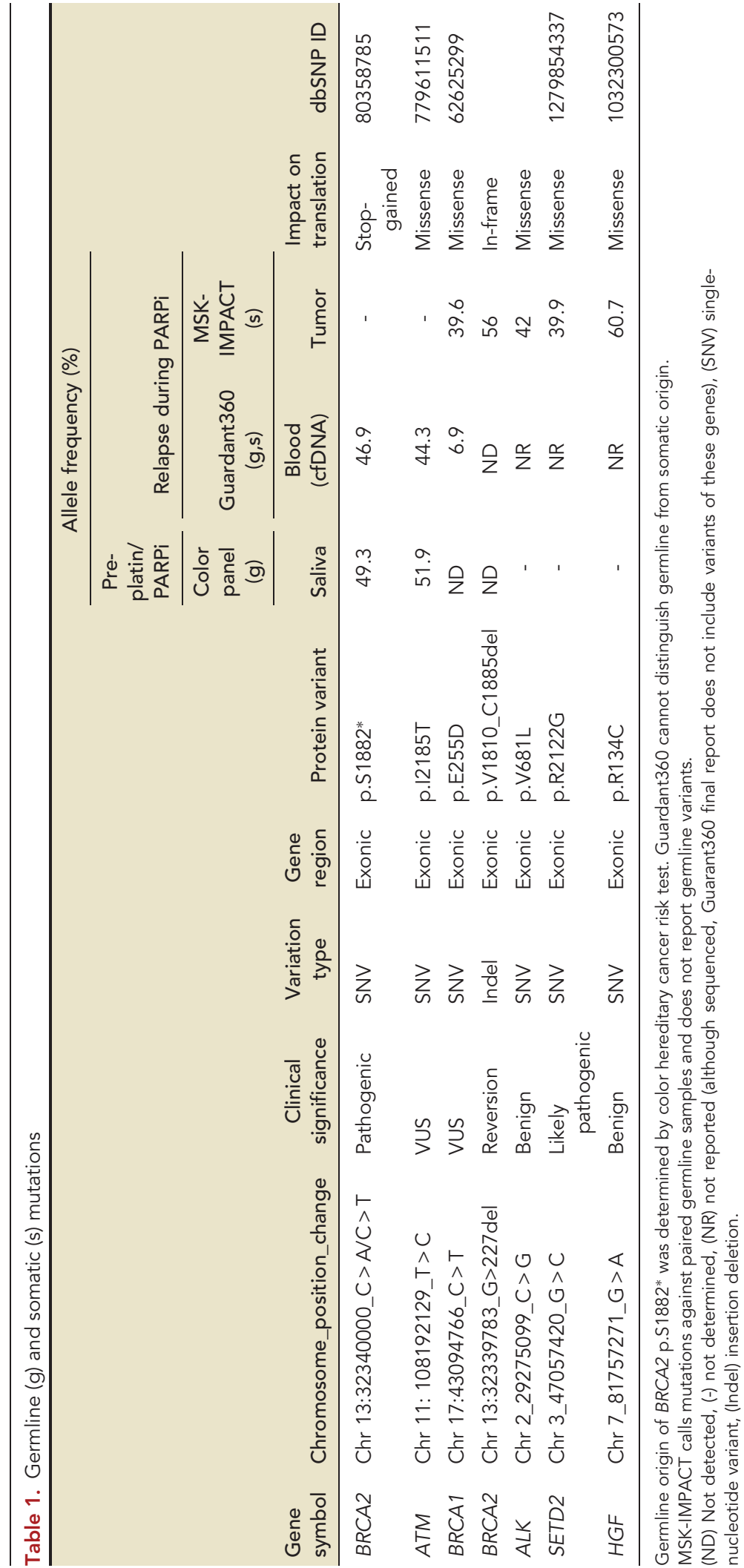




\begin{tabular}{|c|c|c|c|c|c|}
\hline $\begin{array}{l}\text { Gene } \\
\text { symbol }\end{array}$ & Type & Alteration & Location & $\begin{array}{l}\text { Fold } \\
\text { change }\end{array}$ & Panel \\
\hline PTEN & Whole gene & Deletion & $10 q 23.31$ & -3.2 & MSK-IMPACT \\
\hline TNFRSF14 & Whole gene & Loss & $1 p 36.32$ & -1.8 & \\
\hline HNF1A & Whole gene & Loss & $12 q 24.31$ & -1.7 & \\
\hline PIK3CD & Whole gene & Loss & $1 \mathrm{p} 36.22$ & -1.8 & \\
\hline PDCD1 & Whole gene & Loss & $2 q 37.3$ & -1.7 & \\
\hline SETD8 & Whole gene & Loss & $12 q 23.31$ & -1.7 & \\
\hline MAP2K1 & Translocation & $\begin{array}{l}\text { t(14;15)(q31.2;q22.31) (Chr } \\
\text { 14:g.84540589::Chr 15: } \\
\text { g.66679738) }\end{array}$ & exon 1 & & \\
\hline $\begin{array}{l}\text { TMPRSS2- } \\
\quad \text { ERG }\end{array}$ & Deletion & $\begin{array}{l}\text { c.56-537:TMPRSS2_c.18 + } \\
\text { 8757:ERGdel }\end{array}$ & $\begin{array}{l}\text { TMPRSS2 exon } \\
1 \text { to ERG } \\
\text { exon } 2\end{array}$ & & \\
\hline КМT2C & Deletion & c. $9453+378 \_c .11536 \mathrm{del}$ & Exons 41-44 & & \\
\hline
\end{tabular}

Amplification is considered when there is a greater than twofold change detected and deletion when $<-2$ of fold change is detected.

translocation is present in approximately half of prostate cancers (Mosquera et al. 2009), Additional findings included a tumor mutation burden (TMB) of 4.4 mutations per megabase and microsatellite instability (MSI) status stable.

The patient's somatic BRCA2 (V1810_C1885del) mutation is a 228-bp deletion that includes the germline deleterious point mutation (S1882*) at the third to the last position, restoring the open reading frame, albeit a truncated predicted protein (Fig. 3A). This novel mutation could be considered a reversion mutation, if occurring in cis with the germline mutation. It has not been reported in the IRC Database of Reversion Mutations (https ://reversions.icr.ac.uk/), one of the largest of such databases (Pettitt et al. 2020). Because BRCA2 allelic loss was not reported by MSK-IMPACT, we attempted to determine this by performing qualitative polymerase chain reaction ( $\mathrm{PPCR}$ ) and Sanger sequencing on microdissected tumor tissue (Fig. 3B,C). Gel electrophoresis of the PCR products from BRCA2 mutation-specific primers revealed the presence of two distinct bands migrating at 230 and 450 bp. Sanger sequencing of each gel-isolated and -purified band confirmed the secondary deletion mutation but also revealed two electropherogram peaks for the wild-type and germline mutant alleles. TaqMan qPCR genotyping assay was performed to target the germline point mutation but showed the same heterogeneity of wild-type and mutant alleles; this was in part due to the inability to eliminate all normal cells from the biopsy samples. Although we could not confirm that the somatic BRCA2 deletion was cis to the germline mutation, the concurrent emergence of resistance to platinum and PARPi therapies as well as several lines of evidence support this as a reversion mutation: (1) The affected region is frequently deleted in reversions; (2) the deletion includes the germline mutation and restores the open reading frame (ORF), an event unlikely to occur at the same location on the normal allele; and (3) the deletion is flanked by microhomology (CAA), a characteristic of many reversion mutations (Pettitt et al. 2020).

Although the Guardant360 assay did not detect our patient's deletion (cfDNA fragments in the assay are in general 150 bp in length), by using deletion targeted primers on ctDNA taken at a later time, we were able to detect the deletion mutation from a blood sample (see Supplemental Fig. 1). 
A

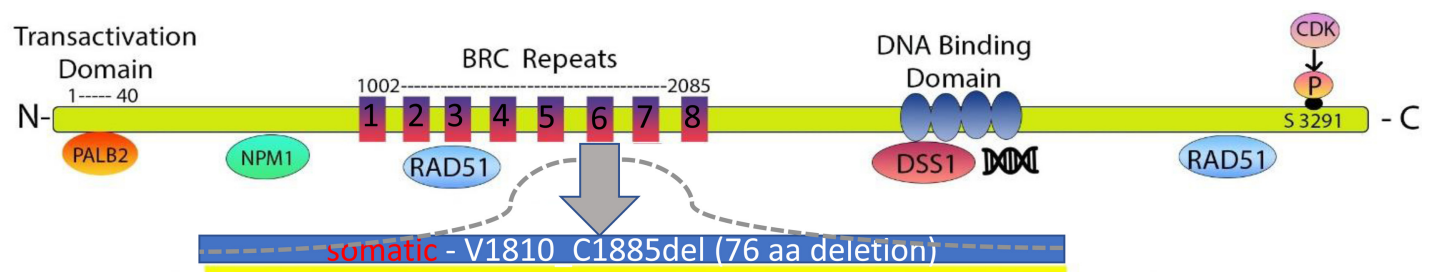

----QTVNEDIC VEELVTSSSPCKNKNAAIKLSISNSNNFEVGPPAFRIASGKIVCVSHETIKKVKDIFTDSFSKVIKENNENKSKIC QTKIMAG---

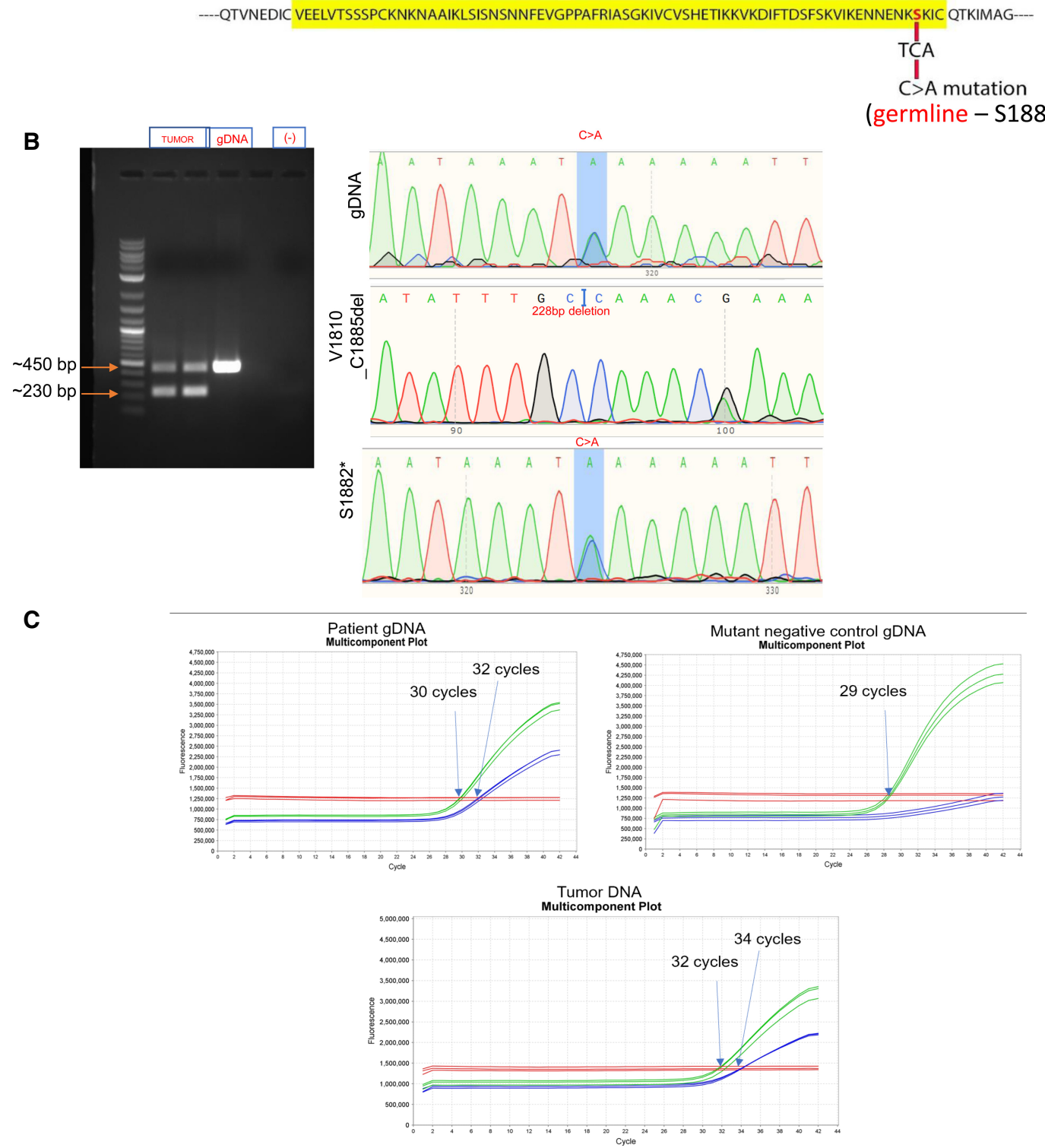

FAM $\square$ - mutation, VIC $\square$ - wild-type, ROX $\square$ - reference

Figure 3. Detection of case patient BRCA2 germline and somatic mutations in different tissue compartments. (A) BRCA2 domains showing location of patient's germline and somatic mutations. The patient has a germline mutation S1882*, which is a pathogenic nonsense mutation. During PARPi (olaparib) treatment, a somatic deletion mutation (V1810_C1885del) of 76 aa was detected. To confirm reversion status of the deletion mutant, Sanger and GPCR (B) were performed. As shown in B, gel electrophoresis (left) from the PCR performed on the primers designed outside the deleted region showed two clear bands of $230 \mathrm{bp}$ and $458 \mathrm{bp}$ and Sanger sequencing (right) on each of these bands confirmed the somatic deletion mutation but also showed two peaks, for the wild-type and germline point mutation. To further confirm these findings, we designed a TaqMan genotyping assay for the point mutation and performed qPCR $(C)$. GPCR on gDNA showed the wild-type and point mutation alleles at 30 and 32 cycles, respectively. QPCR performed on PARPi resistant tumor tissue also detected both alleles. 


\section{DISCUSSION}

The optimal management of individuals with incurable malignancies characterized by HRR deficiency is still evolving. Although germline mutations in BRCA1/2 and PALB2 predict response to both platinum-based chemotherapy and PARP inhibition, because of the requirement to repair double-strand DNA breaks through the error-prone, nonhomologous end joining pathway, the application of these therapies appears to be tumor-specific (Tutt et al. 2005). For example, platinum induction followed by PARP inhibitor maintenance has become a standard of care in the treatment of ovarian and pancreatic cancers with gBRCA1/2 or PALB2 mutations (Robson et al. 2017; Golan et al. 2019). In gBRCA1/2-mutated metastatic breast cancer, however, whereas platinum chemotherapy is being utilized in the first-line setting, PARP inhibitors are reserved for relapsed disease (Moore et al. 2018). The use of these genome-directed therapies is inherently more complicated in prostate cancer, which encompasses both castration-sensitive and castration-resistant adenocarcinomas as well as treatment-emergent and de novo neuroendocrine carcinomas.

Prostate adenocarcinoma with a gBRCA1/2 mutation is associated with an aggressive clinical course and poor prognosis compared to nonmutated disease (Castro et al. 2013). Platinum-based chemotherapy has not been prospectively studied in HRR-deficient prostate cancer and there is no general consensus on whether it is more useful than standard therapies (Pomerantz et al. 2017; Mateo et al. 2018). On the other hand, the PARP inhibitors olaparib and rucaparib have both recently been approved by the U.S. Food and Drug Administration for use in recurrent CRPC with germline or somatic HRR gene mutations.

Neuroendocrine prostate cancer (NEPC) has traditionally been considered a rare, lethal subtype of prostate cancer (Akamatsu et al. 2018). Yet, the widespread use of and subsequent resistance to newer, potent anti-androgen drugs is increasing the incidence of NEPC, specifically t-SCNC (Aggarwal et al. 2018). This observation, coupled with an expansion in germline genetic testing of individuals with prostate cancer, will likely result in more oncologists encountering t-SCNC with HRR gene mutations. Although platinum-based chemotherapy has demonstrated efficacy as first-line therapy, there are no clinical trial results on the use of PARP inhibitors for relapsed disease or as maintenance therapy. Therefore, case reports and series are important in informing practice decisions until mature clinical trial data are reported.

To our knowledge, this is the first case report of germline BRCA2-mutated t-SCNC of the prostate. Our patient developed explosive t-SCNC after 16 mo of ADT plus abiraterone for Gleason 8 metastatic prostate cancer. He achieved a complete response with first-line platinum-based chemotherapy but a $<6$-mo remission duration, despite the use of a PARP inhibitor as maintenance therapy. Given this brief remission, which includes a recognized continued benefit of chemotherapy for $\sim 2$ mo after its completion, the benefit of olaparib was considered brief at best. The mechanism of PARP inhibitor resistance in our patient was revealed by tumor genomic profiling, which found a somatic BRCA2 mutation. This BRCA2 (V1810_C1885del) deletion mutant encodes for nearly full-length BRCA2 protein except for a deletion of the BRC repeat 6 . This would most likely restore at least partial wildtype function as the other intact BRC repeats would allow for the necessary stabilization of RAD51-ssDNA interactions (Chatterjee et al. 2016; A. Monteiro pers. commun.). In support of this, the IRC Database of Reversion Mutations documents a case of platinum-resistant ovarian cancer with a BRCA2 germline 5946delT reverting to a functional allele by deleting c.5954-6090 (aa 1977-2002), which is part of the BRC repeat 7 (Edwards et al. 2008).

The case presented also has clinical relevance regarding the uses of cfDNA analysis vs tumor profiling to detect BRCA2 reversion mutations in patients with prostate cancer. The lack of detection of our reversion mutation by Guardant360 may relate to its large size (228 bp) relative to the typical reversion mutation, which is <100 bp (Pettitt et al. 2020). 
Although some groups recommend cfDNA analysis over tumor biopsy for detection of the full spectrum of tumor heterogeneity (Carneiro et al. 2018), others have found that the assay has limitations in detecting clinically relevant alterations (including large somatic deletions) in a significant percentage of patients (Taavitsainen et al. 2019).

Both epigenetic and genetic changes have been linked to the lineage plasticity of prostate cancer (Ge et al. 2020). Altered transcriptional regulation by DNA methylation, histone modification, and chromatin remodeling plays a prominent role in the clonal evolution from hormone-sensitive adenocarcinoma to castration-resistant neuroendocrine cancer (Beltran et al. 2016). In our patient, we observed somatically acquired alterations in three histone lysine N-methyltransferase genes, including a missense mutation of SETD2, whole-gene loss of SETD8 (KMT5A), and deletion in KMT2C. It has recently been shown that SETD2-mediated H3K36me3 down-regulates EZH2-catalyzed H3K27me3 chromatin repression and that SETD2 deficiency promotes the development of metastatic prostate cancer (Yuan et al. 2020). Similarly, SETD8 reduces EZH2-mediated cell proliferation, and reduction in SETD8 activity promotes oncogenesis (LeFave et al. 2015).

Our patient's genomic profile revealed a mutation in the PTEN gene and a TMPRSS2ERG fusion, which are present in $~ 50 \%$ of prostate cancers (Akamatsu et al. 2018). Yet, we did not detect loss of RB1 and TP53 nor gene amplification of MYCN or AURKA, common alterations in NEPC (Beltran et al. 2011; Ge et al. 2020). We did not test for MYCN and AURKA overexpression by immunohistochemistry, however, and therefore cannot rule out their involvement in this case of t-SCNC.

Another case of BRCA2-alterered t-SCNC was recently reported by Turina et al. (2019). In contrast to our patient, their patient did not have a gBRCA2 mutation but rather somatic complete copy-number loss of BRCA2 found on tumor genomic profiling. The patient developed t-SCNC after 4 mo of ADT plus enzalutamide, had a complete response to etoposide/ carboplatinum, and was in continued remission after 9 mo of olaparib; no further clinical detail was provided. Considering both cases, inactivation of $B R C A 2$, whether through germline or somatic alteration, appears to render t-SCNC highly sensitive to platinum-based chemotherapy, although resistance to platinum/PARPi occurred within 6 mo in our patient as a result of the emergence of a BRCA2 reversion mutation. Clearly, more reports of HRR-deficient $\mathrm{t}-\mathrm{SCNC}$ are needed for a clearer understanding of the role of PARP inhibitors and the emergence of BRCA2 reversion mutations in response to platinum and/or PARPi therapy in this histologically and genetically defined subtype of prostate cancer.

In summary, the case presented provides evidence for initial platinum sensitivity of gBRCA2-mutated t-SCNC and emergence of a BRCA2 somatic reversion mutation during PARPi maintenance therapy. The candidate reversion mutation was detectable through tumor genomic profiling but not routine cfDNA analysis. PARP inhibition following effective platinum-based chemotherapy may be a less promising strategy in BRCA2-mutated t-SCNC than in other HRR-deficient malignancies (Robson et al. 2017; Moore et al. 2018; Golan et al. 2019). Clinical trials testing different sequencing of platinum-chemotherapy and PARPi along with both serial cfDNA and tumor sampling next-generation sequencing (NGS) analyses are needed for a more informed treatment paradigm to emerge for the treatment of t-SCNC characterized by mutations in HRR genes.

\section{METHODS}

\section{DNA Isolation and PCR Assay Design}

Total genomic DNA isolation was performed from formalin-fixed paraffin-embedded (FFPE) tumor tissue sample (collected after PARPi treatment) by GeneRead DNA FFPE Kit (QIAGEN 180134) and from peripheral blood monocytes by Gentra Puregene Blood Kit (QIAGEN 
$8_{\text {CSH }}^{\infty}$ C O L D S P R IN G H A R B OR Molecular Case Studies
Neuroendocrine prostate cancer with BRCA mutation
Competing Interest Statement

The authors have declared no competing interest.

\section{Referees}

Michael Fraser

Anonymous

Received August 10, 2020; accepted in revised form January 6, 2021.
158422). Double-strand DNA concentrations were determined by Qubit 2.0 Fluorometer (Invitrogen by Life Technologies). A primer pair (forward: CTTGATTCTGGTATTGA GCCAGT, reverse: ACCTTATGTGAATGCGTGCT) was designed to the BRCA2 gene exon 11 to sequence somatic mutation as well as point mutation. PCR product DNAs were isolated from the agarose gel by QIAquick Gel Extraction Kit (QIAGEN 28704) and sent for Sanger sequencing (Genewiz). For the mutation analysis by qPCR, TaqMan SNP Genotyping Assay (ThermoFisher Scientific) was designed for point mutation (S1882*) and qPCR was performed on QuantStudio 7 Flex (ThermoFisher Scientific).

\section{ADDITIONAL INFORMATION}

\section{Data Deposition and Access}

Two BRCA2 variants (S1882*, V1810_C1885del) focused on in this report have been submitted to ClinVar (https://www.ncbi.nlm.nih.gov/clinvar/) and can be found under accession numbers SCV001451935 and SCV001451938, for S1882* and V1810_C1 885del, respectively.

\section{Ethics Statement}

Informed written consent was obtained for the patient for the study, Genomic Profiling in Cancer Patients (Memorial Sloan Kettering Cancer Center IRB, ClinicalTrials.gov ID: NCT01775072).

\section{Acknowledgments}

We thank Dr. Alvaro N. Monteiro (H. Lee Moffitt Cancer Center \& Research Institute) and Dr. Stephen J. Pettit and Dr. Christopher J. Lord (Institute of Cancer Research), for their expertise and generous advice. We also thank Dr. Joanna Weber for assistance with histopathology, Dr. Sabina Swierczek for assistance with Sanger and qPCR experiments, and Dr. Sandra Lobo for support and encouragement.

\section{REFERENCES}

Aggarwal R, Huang J, Alumkal JJ, Zhang L, Feng FY, Thomas GV, Weinstein AS, Friedl V, Zhang C, Witte ON, et al. 2018. Clinical and genomic characterization of treatment-emergent small-cell neuroendocrine prostate cancer: a multi-institutional prospective study. J Clin Onco/ 36: 2492-2503. doi:10.1200/JCO.2017.77 .6880

Akamatsu S, Inoue T, Ogawa O, Gleave ME. 2018. Clinical and molecular features of treatment-related neuroendocrine prostate cancer. Int J Urol 25: 345-351. doi:10.1111/iju.13526

Antonarakis ES, Piulats JM, Gross-Goupil M, Goh J, Ojamaa K, Hoimes CJ, Vaishampayan U, Berger R, Sezer A, Alanko T, et al. 2020. Pembrolizumab for treatment-refractory metastatic castration-resistant prostate cancer: multicohort, open-label phase II KEYNOTE-199 study. J Clin Onco/ 38: 395-405. doi:10.1200/JCO.19 .01638

Apostolidis L, Nientiedt C, Winkler EC, Berger AK, Kratochwil C, Kaiser A, Becker AS, Jager D, Hohenfellner M, Huttenbrink C, et al. 2019. Clinical characteristics, treatment outcomes and potential novel therapeutic options for patients with neuroendocrine carcinoma of the prostate. Oncotarget 10: 17-29. doi:10.18632/ oncotarget.26523

Beltran H, Rickman DS, Park K, Chae SS, Sboner A, MacDonald TY, Wang Y, Sheikh KL, Terry S, Tagawa ST, et al. 2011. Molecular characterization of neutroendocrine prostate cancer and identification of new drug targets. Cancer Discov 1: 487-495. doi:10.1158/2159-8290.CD-11-0130

Beltran H, Prandi D, Mosquera JM, Benelli M, Puca L, Cytra J, Marotz C, Giannopoulou E, Chakravarthi B, Varambally S, et al. 2016. Divergent clonal evolution of castration-resistant neuroendocrine prostate cancer. Nat Med 22: 298-305. doi:10.1038/nm.4045 
Carneiro BA, Collier KA, Nagy RJ, Pamarthy S, Sagar V, Fairclough S, Odegaard J, Lanman RB, Costa R, Taxter $T$, et al. 2018. Acquired resistance to Poly (ADP-ribose) polymerase inhibitor olaparib in BRCA2-associated prostate cancer resulting from biallelic BRCA2 reversion mutations restores both germline and somatic loss-of-function mutations. JCO Precis Oncol 2: 1-8. doi:10.1200/PO.17.00176

Castro E, Goh C, Olmos D, Saunders E, Leongamornlert D, Tymrakiewicz M, Mahmud N, Dadaev T, Govindasami K, Guy M, et al. 2013. Germline BRCA mutations are associated with higher risk of nodal involvement, distant metastasis, and poor survival outcomes in prostate cancer. J Clin Onco/ 31: 1748-1757. doi:10.1200/JCO.2012.43.1882

Chatterjee G, Jimenez-Sainz J, Presti T, Nguyen T, Jensen RB. 2016. Distinct binding of BRCA2 BRC repeats to RAD51 generates differential DNA damage sensitivity. Nucleic Acids Res 44: 5256-5270. doi:10.1093/nar/ gkw242

Corn PG, Heath El, Zurita A, Ramesh N, Xiao L, Sei E, Li-Ning-Tapia E, Tu SM, Subudhi SK, Wang J, et al. 2019. Cabazitaxel plus carboplatin for the treatment of men with metastatic castration-resistant prostate cancers: a randomised, open-label, phase 1-2 trial. Lancet Oncol 20: 1432-1443. doi:10.1016/S1470-2045(19) 30408-5

de Bono J, Mateo J, Fizazi K, Saad F, Shore N, Sandhu S, Chi KN, Sartor O, Agarwal N, Olmos D, et al. 2020. Olaparib for metastatic castration-resistant prostate cancer. N Engl J Med 382: 2091-2102. doi:10.1056/ NEJMoa1911440

Edwards SL, Brough R, Lord CL, Natarajan R, Vatcheva R, Levine DA, Boyd J, Reis-Filho JS, Ashworth A. 2008. Resistance to therapy caused by intragenic deletion in BRCA2. Nature 451: 1111-1115. doi:10.1038/ nature06548

Ge R, Wang Z, Montironi R, Jiang Z, Cheng M, Santoni M, Huang K, Massari F, Lu X, Cimadamore A, et al. 2020. Epigenetic modulations and lineage plasticity in advanced prostate cancer. Ann Oncol 31: 470-478. doi:10.1016/j.annonc.2020.02.002

Golan T, Hammel P, Reni M, Van Cutsem E, Macarulla T, Hall MJ, Park JO, Hochhauser D, Arnold D, Oh DY, et al. 2019. Maintenance olaparib for germline BRCA-mutated metastatic pancreatic cancer. N Engl J Med 381: 317-327. doi:10.1056/NEJMoa1903387

LaFave LM, Béguelin W, Koche R, Teater M, Spitzer B, Chramiec A, Papalexi E, Keller M, Hricik T, Konstantinoff K, et al. 2015. Loss of BAP1 function leads to EZH2-dependent transformation. Nat Med 21: 1344-1349.

Mateo J, Cheng HH, Beltran H, Dolling D, Xu W, Pritchard CC, Mossop H, Rescigno P, Perez-Lopez R, Sailer V, et al. 2018. Clinical outcome of prostate cancer patients with germline DNA repair mutations: retrospective analysis from an international study. Eur Urol 73: 687-693. doi:10.1016/j.eururo.2018.01.010

Moore K, Colombo N, Scambia G, Kim BG, Oaknin A, Friedlander M, Lisyanskaya A, Floquet A, Leary A, Sonke GS, et al. 2018. Maintenance olaparib in patients with newly diagnosed advanced ovarian cancer. N Engl J Med 379: 2495-2505. doi:10.1056/NEJMoa1810858

Mosquera JM, Mehra R, Regan MM, Perner S, Genega EM, Bueti G, Shah RB, Gaston S, Tomlins SA, Wei JT, et al. 2009. Prevalence of TMPRSS2-ERG fusion prostate cancer among men undergoing prostate biopsy in the United States. Clin Cancer Res 15: 4706-4711. doi:10.1158/1078-0432.CCR-082927

Pettitt SJ, Frankum JR, Punta M, Lise S, Alexander J, Chen Y, Yap TA, Haider S, Tutt ANJ, Lord CJ. 2020. Clinical BRCA1/2 reversion analysis identifies hotspot mutations and predicted neoantigens associated with therapy resistance. Cancer Discov 10: 1475-1488. doi:10.1158/2159-8290.CD-191485

Pomerantz MM, Spisak S, Jia L, Cronin AM, Csabai I, Ledet E, Sartor AO, Rainville I, O'Connor EP, Herbert $\mathrm{ZT}$, et al. 2017. The association between germline BRCA2 variants and sensitivity to platinum-based chemotherapy among men with metastatic prostate cancer. Cancer 123: 3532-3539. doi:10.1002/cncr .30808

Robson M, Im SA, Senkus E, Xu B, Domchek SM, Masuda N, Delaloge S, Li W, Tung N, Armstrong A, et al. 2017. Olaparib for metastatic breast cancer in patients with a germline BRCA mutation. N Engl J Med 377: 523-533. doi:10.1056/NEJMoa1706450

Sartor O, de Bono JS. 2018. Metastatic prostate cancer. N Engl J Med 378: 645-657. doi:10.1056/ NEJMra1701695

Swami U, McFarland TR, Nussenzveig R, Agarwal N. 2020. Advanced prostate cancer: treatment advances and future directions. Trends Cancer 6: 702-715. doi:10.1016/j.trecan.2020.04.010

Taavitsainen S, Annala M, Ledet E, Beja K, Miller PJ, Moses M, Nykter M, Chi KN, Sartor O, Wyatt AW. 2019. Evaluation of commercial circulating tumor DNA test in metastatic prostate cancer. JCO Precis Oncol 3: 19. doi:10.1200/PO.19.00014

Turina CB, Coleman DJ, Thomas GV, Fung AW, Alumkal JJ. 2019. Molecular testing identifies determinants of exceptional response and guides precision therapy in a patient with lethal, treatment-emergent neuroendocrine prostate cancer. Cureus 11: e5197. doi:10.7759/cureus.5197 
Tutt AN, Lord CJ, McCabe N, Farmer H, Turner N, Martin NM, Jackson SP, Smith GC, Ashworth A. 2005. Exploiting the DNA repair defect in BRCA mutant cells in the design of new therapeutic strategies for cancer. Cold Spring Harb Symp Quant Biol 70: 139-148. doi:10.1101/sqb.2005.70.012

Wang HT, Yao YH, Li BG, Tang Y, Chang JW, Zhang J. 2014. Neuroendocrine prostate cancer (NEPC) progressing from conventional prostatic adenocarcinoma: factors associated with time to development of NEPC and survival from NEPC diagnosis-a systematic review and pooled analysis. J Clin Oncol 32: 3383-3390. doi:10.1200/JCO.2013.54.3553

Yuan H, Han Y, Wang X, Li N, Liu Q, Yin Y, Wang H, Pan L, Li L, Song K, et al. 2020. SETD2 restricts prostate cancer metastasis by integrating EZH2 and AMPK signaling pathways. Cancer Cell 38: 350-365.

Zehir A, Benayed R, Shah RH, Syed A, Middha S, Kim HR, Srinivasan P, Gao J, Chakravarty D, Devlin SM, et al. 2017. Mutational landscape of metastatic cancer revealed from prospective clinical sequencing of 10,000 patients. Nat Med 23: 703-713. doi:10.1038/nm.4333 


\section{COLD SPRING HARBOR Molecular Case Studies}

\section{Treatment-emergent neuroendocrine prostate cancer with a germline BRCA2 mutation: identification of a candidate reversion mutation associated with platinum/PARP-inhibitor resistance}

Deep Pandya, Myra Shah, Fuat Kaplan, et al.

Cold Spring Harb Mol Case Stud 2021, 7: a005801

Access the most recent version at doi: $10.1101 / \mathrm{mcs} . a 005801$

Supplementary http://molecularcasestudies.cshlp.org/content/suppl/2021/02/18/mcs.a005801.D
Material

References This article cites 29 articles, 5 of which can be accessed free at: http://molecularcasestudies.cshlp.org/content/7/1/a005801.full.html\#ref-list-1

License This article is distributed under the terms of the Creative Commons Attribution-NonCommercial License, which permits reuse and redistribution, except for commercial purposes, provided that the original author and source are credited.

Email Alerting Receive free email alerts when new articles cite this article - sign up in the box at the Service top right corner of the article or click here. 